\title{
Outbreak of invasive pneumococcal disease at a Belfast shipyard in men exposed to welding fumes, Northern Ireland, April-May 2015: preliminary report
}

L Patterson ${ }^{1,2}$, N Irvine², A Wilson², L Doherty², A Loughrey³, L Jessop (lucy.jessop@hscni.net)2

1. UK Field Epidemiology Training Programme, Public Health England, United Kingdom

2. Public Health Agency, Health Protection Service, Belfast, Northern Ireland

3. Belfast Health and Social Care Trust, Royal Victoria Hospital, Belfast, Northern Ireland

Citation style for this article:

Patterson L, Irvine N, Wilson A, Doherty L, Loughrey A, Jessop L. Outbreak of invasive pneumococcal disease at a Belfast shipyard in men exposed to welding fumes, Northern Ireland, April-May 2015: preliminary report. Euro Surveill. 2015;20(21):pii=21138. Available online: http://www.eurosurveillance.org/ViewArticle. aspx?Articleld $=21138$

Article submitted on 20 May 2015 / published on 28 May 2015

We report an outbreak of four confirmed cases of invasive pneumococcal disease (IPD) in individuals occupationally exposed to welding fumes, at a Belfast shipyard (Northern Ireland). All cases were hospitalised. A high-risk sub-group of 679 workers has been targeted for antibiotic prophylaxis and pneumococcal vaccination. Physicians and public health institutions outside Northern Ireland should be alert to individuals presenting with pneumonia or IPD and recent links to the shipyard, to facilitate early assessment and treatment.

\section{Incident description}

On 29 April 2015, two cases of invasive pneumococcal disease (IPD) with dates of onset of illness on 28 April were notified to the regional Health Protection Service, Public Health Agency (PHA) Northern Ireland. The only epidemiological link that could be identified was that they both worked on the refurbishment of an oil rig at a Belfast shipyard. PHA signposted the shipyard to the United Kingdom (UK) Health and Safety Executive (HSE) guidelines and reinforced the importance of personal protective equipment (PPE). The isolates were sent for typing to the Public Health England (PHE) Respiratory and vaccine preventable bacteria reference unit (RVPBRU). On 13 May, the PHA was notified of two further cases of IPD with onset dates on 6 May, in men who worked at the same shipyard.

The cases were in men aged in their 205 to 50 from the UK and two other European countries. All were from occupational groups potentially exposed to welding fumes. All presented with pneumonia, and were microbiologically confirmed by isolation of Streptococcus pneumoniae from blood cultures (three cases) or detection of urinary antigen (one case). All isolates were fully sensitive to amoxicillin and erythromycin. Typing of all three blood culture isolates revealed serotype 4; the urinary antigen positive sample could not be typed.

\section{Shipyard workforce}

Up to 1,500 individuals can be on site with a multinational staffing component including for example Bulgarian, Lithuanian, Norwegian, Polish, Romanian and Russian nationalities (approximately one third of all staff). The workforce consists of core workers and temporary / agency staff who are employed by a range of contractors, themselves also based in different European countries. The obligation for occupational health provision lies with the contractors for their respective workers.

\section{Epidemiological investigations}

We are using a specific case definition for the purpose of the outbreak investigation and active case finding. A confirmed case is an individual who has worked at the Belfast shipyard since mid-January 2015 with a clinical diagnosis of IPD or pneumococcal pneumonia AND at least one of the following: pneumococcus isolated from normally sterile site (blood, cerebrospinal fluid (CSF), joint, peritoneum, pleural fluid or other, but not sites such as eye), pneumococcal DNA or antigen detected in fluid from a normally sterile site or pneumococcal antigen detected in urine.

We are collecting information on confirmed cases to include patient identifiers and demographic characteristics, risk factors for IPD, clinical details, microbiological investigations, vaccination status and onsite working patterns [1].

Retrospective case finding will be conducted through examination of laboratory-confirmed reports in people aged 18-64 years with specimen dates in 2015 . Prospective case finding is undertaken through local, national and international alerting. Retrospective typing of isolates from IPD cases during 2015 will be carried out to determine circulating pneumococcal serotypes. 
No family members were identified as close contacts in the two days before the onset of symptoms in the cases. Due to the complex working patterns of the cases, no close work contacts, as defined in the Interim UK guidelines for the public health management of clusters of serious pneumococcal disease in closed settings, could be identified [1].

A communication was issued through the European Union early warning and response system (EWRS) on the morning of 15 May to facilitate case finding across Europe.

\section{Background}

Streptococcus pneumoniae is a Gram-positive coccus known to colonise the nasopharyngeal tract and can cause a spectrum of disease such as otitis media, pneumonia and invasive pneumococcal disease [2]. The virulence of the organism is determined by the capsule and over 90 different capsular types (serotypes) have been identified [3]. Clusters of IPD have been reported from closed settings including long-term care facilities, hospitals and households [4]. A number of risk factors have been identified for IPD including extremes of age, certain co-morbidities, smoking and immunosuppression [5]. Welders have an increased risk of IPD, and although not fully understood, this may relate to components of the fumes serving as a nutrient to increase adherence of the pneumococci to the lung tissue or inhalation of the fumes causing damage to the lung's immune defences [5-7]. The UK HSE guidelines recommend that 23-valent pneumococcal polysaccharide vaccine 'should be considered for people whose work exposes them to frequent or continuous exposure to metal fume (e.g. welders), taking into account the exposure control measures in place' [8].

S. pneumoniae isolates from normally sterile sites are reported to the PHA through routine voluntary laboratory reporting arrangements. Based on laboratory reporting, the average background rate of IPD in Northern Ireland (population $1.82 \times 10^{6}$ ) was ca 6 cases per 100,000 population during 2010-2014, with higher rates in the very young and in those aged over 65 years. Not all isolates are routinely serotyped.

\section{Control measures}

A multidisciplinary outbreak control team (OCT) meeting was held on 14 May with a range of local stakeholders, shipyard management and PHE. It was agreed that as no close contacts of the cases could be identified, those groups most exposed to welding fumes would be offered antibiotic prophylaxis with azithromycin or amoxicillin and vaccination with 23-valent pneumococcal polysaccharide vaccine (PPV23) [1,7]. Azithromycin $500 \mathrm{mg}$ once daily for three days was the preferred first-line antibiotic, with a view to optimising compliance. For those with contraindications to azithromycin, amoxicillin $500 \mathrm{mg}$ twice daily for seven days was offered. PPV 23 vaccine was used as the incident was taken as an opportunity to protect this high-risk occupational group not only against serotype 4 , but also against a wider range of serotypes which might be encountered in future [9].

Antibiotic prophylaxis and vaccination was offered to anyone who was considered at risk of contracting IPD, i.e. worked as a welder or in other occupations with prolonged exposure to metal fumes in enclosed spaces in the working environment. A total of 679 among the around 1,500 shipyard workers met this definition and attended the clinics for the intervention between 16 and 18 May. Only six of the attendees were aware that they had received the pneumococcal vaccine previously. A multi-disciplinary team of public health and clinical staff, including pharmacists, administrators, translators and shipyard staff delivered the response. Dedicated clinics operated for between eight and eleven hours each day at times appropriate to ensure day and night shift staff had the opportunity to attend.

\section{Conclusion}

This investigation is ongoing and we hope to complete this preliminary information that aims to support our active case finding, with a more complete account, at a later date. A letter has been issued in Northern Ireland to local general practitioners, emergency departments and clinicians to increase awareness of the incident and request that all cases of IPD in shipyard workers are notified to the PHA prospectively.

If public health institutions from other countries are aware of further cases of IPD in individuals who have been working at the Belfast shipyard, Northern Ireland, since the middle of January 2015, we would like to invite them to contact the PHA duty room at pha.dutyroom@ hscni.net in order for us to gain a full overview of the number of cases in this outbreak.

\section{Conflict of interest}

None declared.

\section{Authors' contributions}

LJ led the overall response to the incident and AW is the Port Health lead. Epidemiological investigations were conducted by NI and LP. LD is the Assistant Director for Health Protection and chaired the outbreak control team meetings. Microbiological investigations were conducted by AL. The manuscript was drafted by LP. All authors commented and agreed on the final manuscript.

\section{References}

1. Health Protection Agency (HPA). Interim UK guidelines for the public health management of clusters of serious pneumococcal disease in closed settings. London: HPA. Jul 2008. Available from: https://www.gov.uk/government/uploads/system/ uploads/attachment_data/file/328250/pneumococcal disease_-_July_2008.pdf

2. Steens A, Vestrheim DF, Aaberge IS, Wiklund BS, Storsaeter J, Riise Bergsaker MA, et al. A review of the evidence to inform pneumococcal vaccine recommendations for risk groups aged 2 years and older. Epidemiol Infect. 2014;142(12):2471-82. http:// dx.doi.org/10.1017/S0950268814001514 PMID:24932959 
3. Public Health England (PHE). Immunisation against Infectious Disease and Pneumococcal disease: guidance, data and analysis. Pneumococcal: the green book, chapter 25 .

London: PHE. 2013. [Accessed 14 May 2015]. Available from: https://www.gov.uk/government/uploads/system/uploads/ attachment_data/file/263318/Green-Book-Chapter-25-v5_2.pdf

4. Ihekweazu C, Basarab M, Wilson D, Oliver I, Dance D, George $R$, et al. Outbreaks of serious pneumococcal disease in closed settings in the post-antibiotic era: a systematic review. J Infect. 2010;61(1):21-7. http://dx.doi.org/10.1016/j.jinf.2010.03.032 PMID:20381524

5. Wong A, Marrie TJ, Garg S, Kellner JD, Tyrrell GJ; SPAT Group. Welders are at increased risk for invasive pneumococcal disease. Int J Infect Dis. 2010;14(9):e796-9. http://dx.doi. org/10.1016/j.ijid.2010.02.2268 PMID:20637673

6. Palmer KT, Poole J, Ayres JG, Mann J, Burge PS, Coggon D. Exposure to metal fume and infectious pneumonia. Am J Epidemiol. 2003;157(3):227-33. http://dx.doi.org/10.1093/aje/ kwf188 PMID:12543622

7. Coggon D, Harris EC, Cox V, Palmer KT. Pneumococcal vaccination for welders. Thorax. 2015;70(2):198-9. http:// dx.doi.org/10.1136/thoraxjnl-2014-206129 PMID:25319209

8. Health and Safety Executive (HSE). Pneumonia vaccination for employees exposed to welding and metal fume. 2014. [Accessed 14 May 2015]. Available from: http://www.hse.gov. uk/pubns/eis44.pdf

9. Palmer KT, Cosgrove MP. Vaccinating welders against pneumonia. Occup Med (Lond). 2012;62(5):325-30. http:// dx.doi.org/10.1093/occmed/kqs055 PMID:22764269 